\title{
Nonextensive aspects of self-organized scale-free gas-like networks
}

\author{
Stefan Thurner 1 * and Constantino Tsallis ${ }^{2,3}$, \\ ${ }^{1}$ Complex Systems Research Group, HNO, Medical University of Vienna, Währinger Gürtel 18-20, A-1090; Austria \\ ${ }^{2}$ Centro Brasileiro de Pesquisas Fisicas, Rua Xavier Sigaud 150 22290-180 Rio de Janeiro-RJ; Brasil \\ ${ }^{3}$ Santa Fe Institute, 1399 Hyde Park, Santa Fe, NM 87501; USA
}

\begin{abstract}
We explore the possibility to interpret as a 'gas' the dynamical self-organized scale-free network recently introduced by Kim et al (2005). The role of 'momentum' of individual nodes is played by the degree of the node, the 'configuration space' (metric defining distance between nodes) being determined by the dynamically evolving adjacency matrix. In a constant-size network process, 'inelastic' interactions occur between pairs of nodes, which are realized by the merger of a pair of two nodes into one. The resulting node possesses the union of all links of the previously separate nodes. We consider chemostat conditions, i.e., for each merger there will be a newly created node which is then linked to the existing network randomly. We also introduce an interaction 'potential' (node-merging probability) which decays with distance $d_{i j}$ as $1 / d_{i j}^{\alpha}(\alpha \geq 0)$. We numerically exhibit that this system exhibits nonextensive statistics in the degree distribution, and calculate how the entropic index $q$ depends on $\alpha$. The particular cases $\alpha=0$ and $\alpha \rightarrow \infty$ recover the two models introduced by Kim et al.
\end{abstract}

PACS numbers: $\quad$ 05.70.Ln, 89.75.Hc, 89.75.-k

\section{INTRODUCTION}

A common feature in several recent approaches to complex networks with statistical mechanical methods is the definition of network Hamiltonians [1, 2, 3]. Such Hamiltonians currently depend on the number of links either on a global level, or on the degree of individual nodes. This definition allows to borrow powerful concepts from statistical physics such as the maximum entropy principle 1], which may provide the most probable ensembles of networks. Further, Hamiltonians allow to define both thermodynamical ensembles (microcanonical, canonical, grand canonical) 2, 4] and a partition function, which opens the way to compute degree correlation functions in a formalism most familiar to physicists [1, 3].

However, these approaches do not yet aim to explain the structure of degree distributions, and mainly address random networks. A conceptually different approach has been taken recently in [5], where networks are embedded in some metric space and the definition of entropy in networks is broadened. In this work it was noted that the characteristic distribution of the relevant degree of freedom - the degree of nodes - appears to coincide to distribution functions known for nonextensive systems [6, 7]. More precisely, in [5] it was found that, for some preferential attachment network growth models, the resulting degree distributions are of the q-exponential type (defined later on). In the usual preferential attachment model, the probability of a new node (i.e., being added to a network) to attach its link to a pre-existing node $i$ is proportional to this node's number of links, or degree $k_{i}$, i.e., $p_{A} \propto k_{i}$. This is also true for networks embedded in $R^{n}$, where the

*Electronic address: thurner@univie.ac.at

$\dagger$ Electronic address: tsallis@santafe.edu linking probabilities are made dependent on the relative distance of node $i$ to the new node, i.e., $p_{A} \propto k_{i} / d_{i j}^{\alpha}$. Here $\alpha$ is a free parameter that defines the connecting horizon of a new node to the system. For large $\alpha$ the node will link with high probability to a nearby node, whereas distance becomes irrelevant for $\alpha \rightarrow 0$.

A problem which has not yet been explored in the literature is that of a definition of an interaction between nodes, for example in the way one would think of an interaction of gas molecules. In classical statistical mechanics interactions/collisions between gas particles result in a transfer of momentum from one particle to another, under the constraint that momentum is conserved. In elastic interactions this results in a change of direction and speed of particles after a collision, in inelastic ones also in a change of masses of the particles. In this paper we find that the class of self-organizing networks as introduced in [9] opens the possibility to define an 'interaction' between nodes of a network. In analogy to the momentum transfer in the classical situation, in the network case the interactions are defined by a transfer of links. This enables one to think of a network as some sort of gas, which turns out to be describable by distribution functions characteristic of nonextensive statistical mechanics.

\section{MODEL}

Let us consider the following gas-like system. Particles have links among them. The total number of links of a given node is a characteristic quantity of the node, such as the momentum of an ordinary particle. The particles of this 'gas' have no momentum but only their degree. Neither do the particles have an absolute position in space. They possess only a relative distance $d_{i j}$ to each other which is given by the shortest number of links 
between them (sometimes called chemical distance). Particles can interact 'non-elastically'. Upon an interaction one particle ceases to exist and transfers all its links to the other. If the interacting particles $i$ and $j$ have both had links to a third particle $k$ before the interaction, the remaining particle $i$ will keep its link to $k$, while the links of the disappeared particle $j$ to $k$ will be removed, meaning that links are only counted once (and are not weighted). Consider these interactions taking place in a chemostat, such that the number of particles in a closed system is constant. This means that, for every merging interaction, a new particle will be introduced to the system. The interaction is characterized by the probability that two particles meet and transfer links. Given a 'metric' (relative network distance) this probability can be made distance-dependent as in [5]. To do this we introduce a power like potential.

In what follows we numerically study the distribution of the characteristic degree of freedom (the degree of nodes) as a function of the range of the interaction. As in [5], we find that the distribution of this (nonextensive) 'gas' is described by a $q$-exponential.

\section{A. Network dynamics}

There has been a recent suggestion to model scalefree networks of constant size, the so-called self-organized networks [9]. The idea is that at any given timestep one single pair of nodes, say $i$ and $j$, merge together to become one single node. This node keeps the name of one of the original nodes, say for example $i$. This node now gains all the links of the other node $j$, resulting in a change of degree for node $i$ according to

$$
\begin{aligned}
& k_{i} \rightarrow k_{i}+k_{j}-N_{\text {common }}, \text { if }(i, j) \text { not first neighbors } \\
& k_{i} \rightarrow k_{i}+k_{j}-N_{\text {common }}-2 \text {, if }(i, j) \text { first neighbors }
\end{aligned}
$$

where $N_{\text {common }}$ is the number of nodes, which shared links to both of $i$ and $j$ before the merger. In the case that $i$ and $j$ were first neighbors before the merger, i.e., they had been previously linked, the removal of this link will be taken care of by the term -2 in Eq. (11). Next, to keep the system at constant size, a new node is created, and is linked to $r$ randomly chosen nodes from the existing network. Let us note here that the smallest degree found in a network can only be larger or equal to $r$. This will have consequences for the normalization of distribution functions as will be discussed below. In the following (except for Fig. 2) we will restrict ourselves to $r=2$, for simplicity. Thus the smallest degree will always be 2. (This is not at all an important restriction; as an alternative the actual number of links can also be a random number picked, e.g. from a uniform distribution with an average of $\langle r\rangle$, as in [9]). After that we address the next timestep. Nodes in the network start with a small number of links, and gain links through mergerinteractions. Links to the $N_{\text {common }}$ common neighbors of
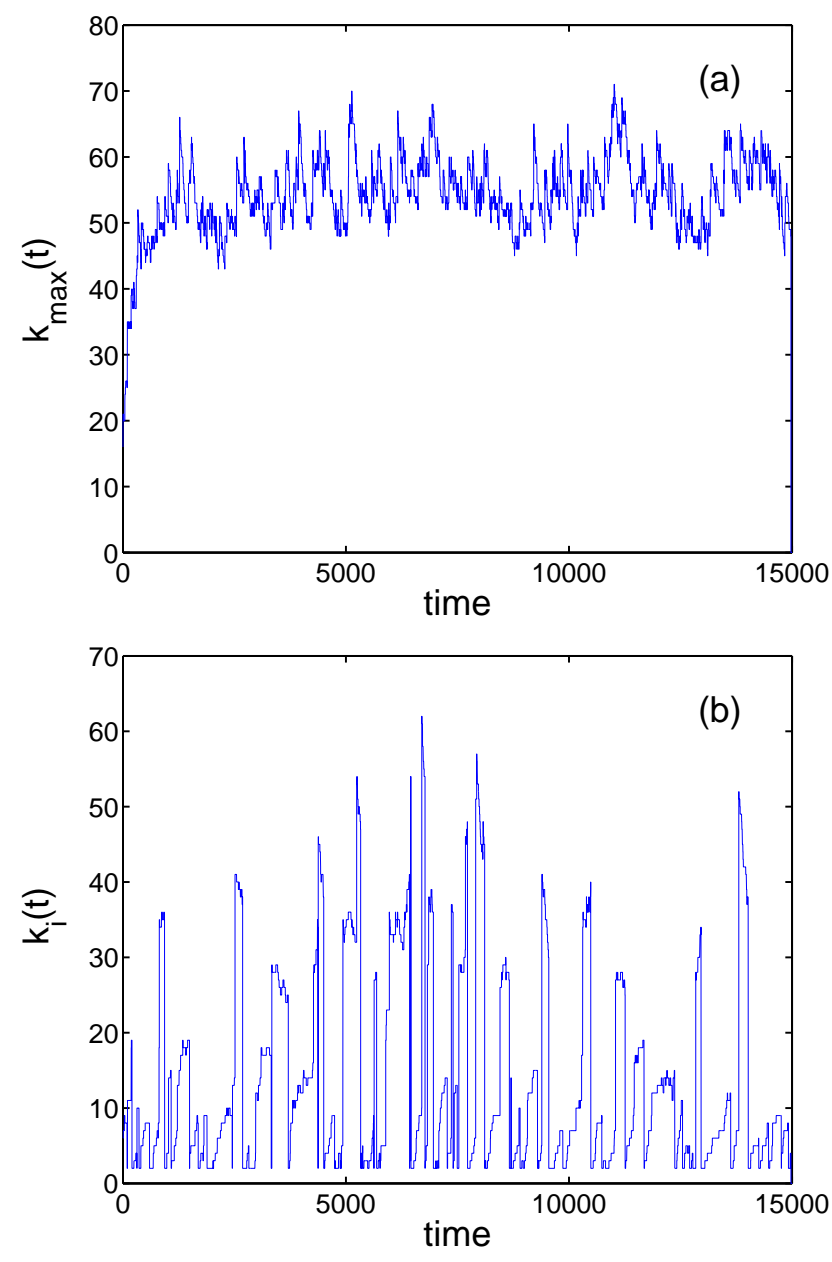

FIG. 1: Simulation of Eq. (1) of a $N=2^{7}$ network with $r=2$ and $\alpha=0$ (random scheme). Time evolution of the degree of (a) the most connected node during the simulation; (b) an individual, randomly chosen node.

two merging nodes are lost as mentioned above, which reduces the number of links. Gains and losses eventually lead to an effective balance over time as shown for instance in Fig. 1 1 a. The number of links of the best connected node in the system is followed over time. After about 1000 timesteps a stationary state is reached. The situation for an individual link is shown in Fig. 1 b. A node starts with $r=2$ links when it is introduced to the system. It gains links through mergers over time. When the node is taken up in a merger it disappears from the system and, as said before, a new one starts with $r=2$ links again. Networks with these rewiring scheme lead to scale-free degree distributions [9], i.e. the power exponent of the cumulative degree distribution tail behaves as, $P(\geq k) \sim k^{-\gamma}$. In [9] two schemes were discussed: The case where only nodes being first-neighbors can merge, and the case where any two nodes - directly connected or not - can merge with the same probability for each possible pair $(i, j)$. The neighbor scheme leads to an ex- 


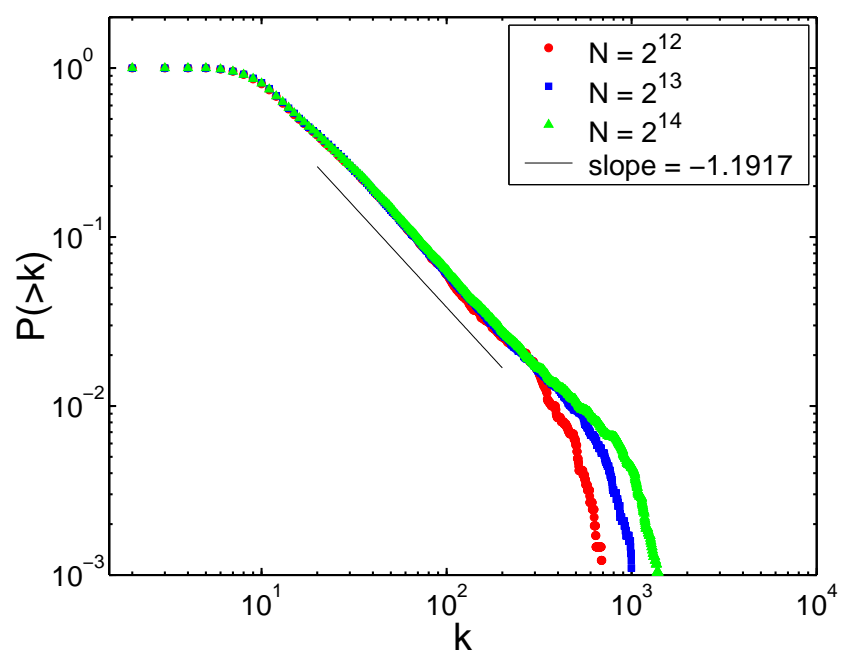

FIG. 2: Cumulative degree distributions for various system sizes $N$ for the neighbor scheme, as reported in [9] with $\langle r\rangle=$ 8. Degrees are from individual states of networks without averaging over identical realizations. On the right side the exponential finite size effect is seen.

ponent $\gamma \sim 1.3$, the random scheme to $\gamma \sim 0.5$. The cumulative degree distribution, for the neighbor scheme is shown in Fig. 2

These distributions can be fit by q-exponentials,

$$
P(\geq k)=e_{q_{c}}^{-(k-2) / \kappa} \quad(k=2,3,4, \ldots),
$$

where the $q$-exponential function is defined, for $1+(1-$ $\left.q_{c}\right) x \geq 0$, as

$$
e_{q_{c}}^{x} \equiv\left[1+\left(1-q_{c}\right) x\right]^{1 /\left(1-q_{c}\right)}
$$

with $\kappa>0$ some characteristic number of links, and $\gamma \equiv 1 /\left(q_{c}-1\right)$ being the tail exponent of the (asymptotic) power-law distribution. Whenever we talk about qvalues corresponding to a cumulative distribution, we use the notation $q_{c}$, where $c$ stands for cumulative. We have normalized with the value corresponding to the smallest possible degree (which in our case equals 2 ) in order to have $P(\geq 2)=1$.

A convenient procedure to perform a two-parameter fit of this kind is to take the $q$-logarithm of the distribution $P$, defined by $Z_{q} \equiv \ln _{q} P(\geq k) \equiv \frac{[P(\geq k)]^{1-q_{c}}-1}{1-q_{c}}$. This is done for a series of different values of $q_{c}$. The function $Z_{q}$ which is best fit with a straight line determines the value of $q_{c}$, the slope being $-\kappa$. The situation for the $N=2^{14}$ data of Fig. 2 is shown in Fig. 3 for $q_{c}$ running between 1 and 2.6.

We numerically verify with good precision that the Ansatz in Eq. (2) for the cumulative degree distribution is a satisfactory one (it could even be the exact one for the present problem). This reveals a connection [5, 10. of scale-free network dynamics to nonextensive statistical mechanics [6, 7]. Let us be more specific. Consider the entropy

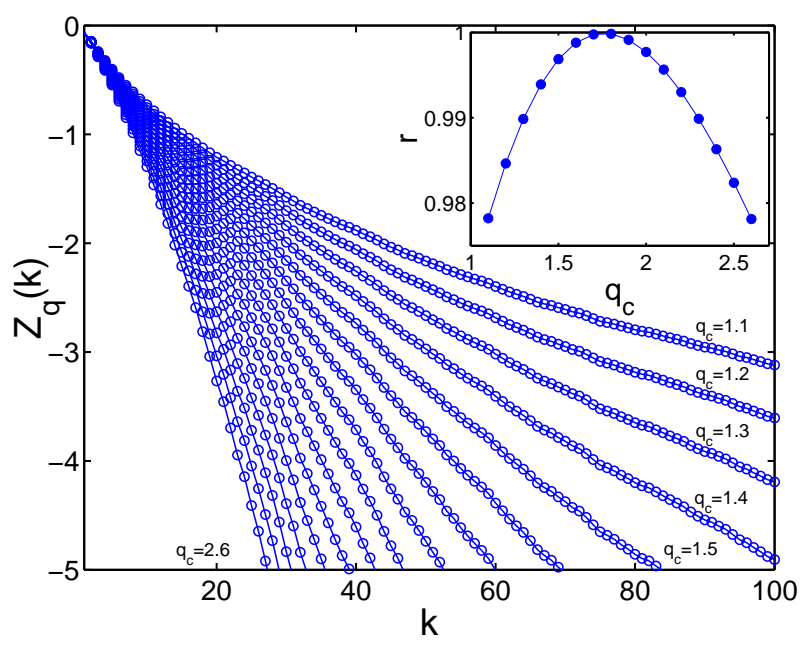

FIG. 3: q-logarithm of the cumulative distribution function from the previous figure as a function of the degree $k$. Clearly, there is a $q_{c}$ which allows for an optimal linear fit. Inset: Linear correlation coefficient $r$ of $Z_{q}(k)$. The value of $q_{c}$ is obtained where $Z_{q}$ is optimally linear, i.e., where the correlation coefficient is maximal. In this example we obtain $q_{c}=1.84$, which corresponds to the slope $\gamma=1.19$ in the previous plot. The continuous curves are guides to the eye.

$$
\begin{aligned}
S_{q} & \equiv \frac{1-\int_{2}^{\infty} d k[p(k)]^{q}}{q-1} \\
{\left[S_{1}\right.} & \left.=S_{B G} \equiv-\int_{2}^{\infty} d k p(k) \ln p(k)\right],
\end{aligned}
$$

where we assume $k$ as a continuous variable for simplicity, and $B G$ stands for Boltzmann-Gibbs. If we extremize $S_{q}$ with the constraints [8]

$$
\int_{2}^{\infty} d k p(k)=1
$$

and

$$
\frac{\int_{2}^{\infty} d k k[p(k)]^{q}}{\int_{2}^{\infty} d k[p(k)]^{q}}=K
$$

we obtain

$$
p(k)=\frac{e_{q}^{-\beta(k-2)}}{\int_{2}^{\infty} d k^{\prime} e_{q}^{-\beta\left(k^{\prime}-2\right)}}=\beta(2-q) e_{q}^{-\beta(k-2)} \quad(k \geq 2),
$$

where $\beta$ is determined through Eq. (6). Both positivity of $p(k)$ and normalization constraint (5) impose $q<2$. The corresponding cumulative distribution $P(>k)$ is then given by

$$
P(>k) \equiv 1-\int_{2}^{k} d k^{\prime} p\left(k^{\prime}\right)=[1-(1-q) \beta(k-2)]^{\frac{2-q}{1-q}} .
$$




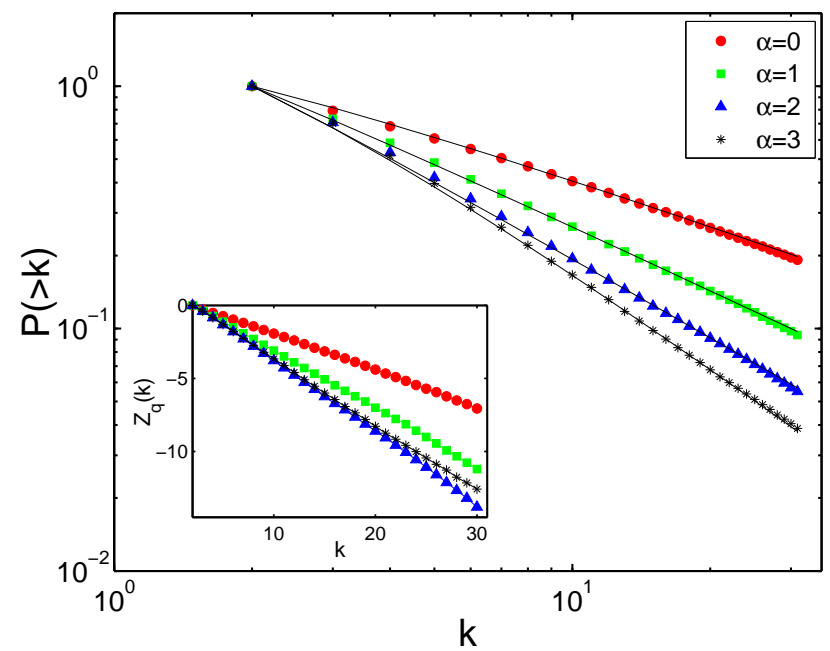

FIG. 4: Cumulative degree distribution for a $N=2^{9}$ network with $r=2$, for typical values of $\alpha$. For $k>30$ finite size effects (such as those illustrated in Fig. 2) emerge. Solid lines are the $q$-exponentials from the fitted parameters $q_{c}$ and $\kappa$, shown in Fig. 5] a. Inset: the same data in q-logarithm vs. linear representation, where we have used, for each value of $\alpha$ the corresponding value of $q_{c}$. For $\alpha$ ranging within the interval $[0,7]$ we verify that the corresponding linear correlation coefficient ranges within the interval [0.999901, 0.999976]. The slope equals $-\kappa$ for each curve (see also inset of Fig. 5 a).

This expression can be rewritten precisely as the Ansatz (2) with

$$
q_{c} \equiv \frac{1}{2-q} ; \kappa \equiv \frac{1}{(2-q) \beta}
$$

\section{B. Network distance and distance-dependent re-linking potential}

Unlike the two schemes in the original presentation of self-organizing networks, the neighbor and the random scheme, we would like to define a distance-dependent merging probability. This needs a definition of distance on the graph. For simplicity we define the distance $d_{i j}$, between two nodes $i$ and $j$ on an undirected graph as the shortest distance, given all links are of unit length. This distance is obtained, for instance, from the Dijkstra algorithm 11]. We randomly choose a node (denoted by $i$ ) and then choose the second node (denoted by $j$ ) of the merger with probability

$$
p_{i j}=\mathcal{N} d_{i j}^{-\alpha} \quad(\alpha \geq 0),
$$

where $\mathcal{N}=1 / \sum_{j} d_{i j}^{-\alpha}$ is a normalization that makes $p_{i j}$ a probability, and $d_{i j}$ is the shortest distance (path) on the network connecting nodes $i$ and $j ; \alpha$ is a real number. Obviously, tuning $\alpha$ from 0 toward large values switches
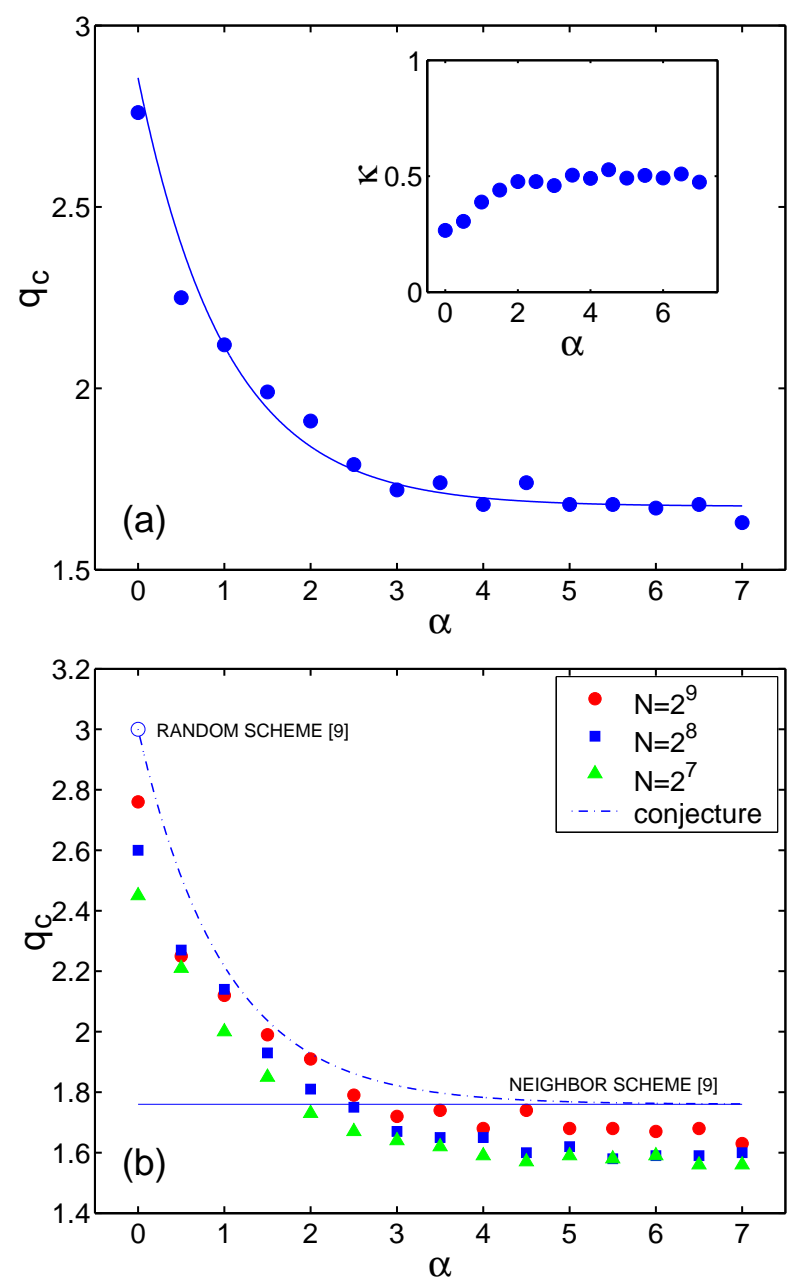

FIG. 5: (a) Dependence of the extensivity parameter $q_{c}$ on the potential parameter $\alpha$ for a $N=2^{9}, r=2$, network. The solid line is an exponential fit to the data points: $q_{c}=$ $1.675+1.1809 e^{-0.985 \alpha}$. We might thus conjecture for the exact answer, the form $q_{c}(\alpha)=q_{c}(\infty)+\left[q_{c}(0)-q_{c}(\infty)\right] e^{-\lambda \alpha}$ with $\lambda \simeq 1$. Inset: $\alpha$-dependence of $\kappa$. (b) Network size dependence of the $q_{c}(\alpha)$ function, for nodes $N=2^{7}, 2^{8}, 2^{9}$. The solid line corresponds to the asymptotic value for large $\alpha$ reported in [9] (neighbor scheme), i.e., $q_{c}(\infty)=1.76$. The value for the $\alpha=0$ model as reported in [9] corresponds to $q_{c}(0)=3$ (empty circle). The conjectural form is indicated with $\lambda=1$ (dashed line).

the model from the random to the neighbor scheme in [9].

\section{RESULTS}

Realizing this distance-dependent potential in a numerical simulation we find the degree distributions given in Fig. 4 All following data was obtained from averages over 1000 identical realizations of degree distributions of networks with a number of nodes, $N=2^{9}$. Networks 
have been recorded after 5 network updates. A network update is performed when $N$ mergers have taken place. This corresponds to the $5 \times 2^{9}$ timesteps shown in Fig. 1

From these degree distributions we obtain the index $q_{c}$ and the characteristic degree $\kappa$. Their dependence on $\alpha$ is given in Fig. 5] The $\alpha$-dependence of $q_{c}$ shows the expected tendency. Our result in the limit $\alpha \rightarrow 0$, $q_{c}(0)=2.8$ corresponds to an exponent $\gamma=0.55$, which is about 10 percent lower than the reported value in 9]. The reason for this small discrepancy seems to be a finitesize effect.

To demonstrate that this might indeed be so, in Fig. 5 b we plot the $q_{c}(\alpha)$-dependence for $N=2^{7}, N=2^{8}$ and $2^{9}$ networks for comparison. As network size increases the value of $q_{c}$ approaches the expected value of 3 [9] in the small $\alpha$ limit. For the $\alpha \rightarrow \infty$ limit, the expected value is recovered for the $N=2^{9}$ network, for smaller networks, there is still a visible size effect. This size effect is related to the problem that the finite size cutoff plays a relatively large role, and interferes considerably in the fits in small networks. Simulations on larger networks are certainly desirable, but inaccessible to our present computational power.

\section{DISCUSSION}

To summarize, we have explored the possibility to making some connection between a nonextensive gas and a self-organized scale-free network. We have shown that the characteristic degree distributions are well described by q-exponentials whose parameters vary with the interaction range, i.e. $\alpha$. The limiting cases $\alpha \rightarrow 0$ and $\alpha \rightarrow \infty$ reproduce the situations given in [9], namely the neighbor merging and the random merging.

In the present work we have used the network's intrinsic metric space, i.e., its adjacency matrix, to measure distances $\left(d_{i j}\right)$ between nodes to be merged. This is in variance with what is done in [5], where the network is embedded in a 'geographical' metric space (with distances $r_{i j}$ ). Both models can of course be unified by introducing both merging (with probability $\propto 1 / d_{i j}^{\alpha}$ ) and distance-dependent linking (with probability $\propto 1 / r_{i j}^{\alpha_{A}}$, where $A$ stands for attachment). The degree distribution of such a unified model could still be of the $q$-exponential form with $q_{c}\left(\alpha, \alpha_{A}\right)$. Of course, $q_{c}(\alpha, 0)=q_{c}(\alpha)$ as given in the present paper. It would not be surprising if $q_{c}\left(\alpha, \alpha_{A}\right)$ was a monotonically decreasing function of both variables $\alpha$ and $\alpha_{A}$, with the maximal value being $q_{c}(0,0)$, and with say $q_{c}(\alpha, \infty)=1, \forall \alpha>0$. In such a case, the interval spanned by $q_{c}$ would clearly be wider than that of the present model.

S.T. would like to thank the SFI and in particular J.D. Farmer for their great hospitality and support during Sept-Oct of 2004, when this work was initiated. Support from SI International and AFRL/USA is acknowledged as well.
[1] Park J. and Newman M.E.J., Phys. Rev. E 70, 2004, 066117.

[2] Palla G., Dernyi I., Farkas I. and Vicsek T., Phys. Rev. E 69, 2004, 046117; Farkas I., Dernyi I., Palla G. and Vicsek T., Lect. Notes in Phys. 650, 2004, 163.

[3] Berg J. and Lässig M., Phys. Rev. Lett. 89, 2002, 228701.

[4] Dorogovtsev S.N and Mendes J.F.F., Evolution of Networks: From Biological Nets to the Internet and $W W W$, Oxford University Press, 2003.

[5] Soares D.J.B., Tsallis C., Mariz A.M. and da Silva L.R., Europhys. Lett. 70, 2005, 70.

[6] Tsallis C., J. Stat. Phys 52, 1988, 479.
[7] Gell-Mann M. and Tsallis C., eds., Nonextensive Entropy - Interdisciplinary Applications, Oxford University Press, New York, 2004; Tsallis C., Gell-Mann M. and Sato Y., cond-mat/0502274

[8] Tsallis C., Mendes R.S. and Plastino A.R., Physica A 261, 1998, 534.

[9] Kim B.J., Trusina A., Minnhagen P. and Sneppen K., Eur. Phy. J. B 43, 2005, 369.

[10] Doye J.P.K. and Massen C.P., J. Chem. Phys. 122, 2005, 084105.

[11] Dijkstra E.W., Numerische Mathematik 1, 1959, 269. 